\title{
Caracterização das demandas de psicodiagnóstico infantil em uma clínica-escola de São Paulo
}

\section{Characterization of demand of psychological diagnoses for children at a training clinic in São Paulo \\ Caracterización de las demandas de psicodiagnóstico infantil en una clínica-escuela de São Paulo}

\author{
Lucilena Vagostello* \\ Daiana Santana Monteiro Albuquerque \\ Fernanda Teodoro Queiroz ${ }^{* * * *}$ \\ Gabriela Pacheco Lopes ${ }^{* * * * *}$ \\ Letícia Vieira Silva ${ }^{* * * * *}$
}

\section{Resumo}

O presente estudo objetivou caracterizar a clientela infantil atendida em avaliação psicodiagnóstica, no período de 2007 a 2013, em uma clínica-escola de Psicologia, na cidade de São Paulo. Trata-se de uma pesquisa documental, de natureza quantitativa, realizada por meio de levantamento e análise de livros, fichas de inscrição e prontuários de crianças atendidas. Do total de crianças inscritas ( $N=150$ ), 67,4\% eram do sexo masculino, encaminhados por profissionais da saúde. As queixas escolares foram frequentes em meninos e meninas, juntamente com queixas de reações emocionais às relações familiares.

\footnotetext{
Doutora em Psicologia Clínica pela Universidade de São Paulo, Professora do Curso de Psicologia da Universidade São Judas Tadeu. E-mail: vagostello@yahoo.com.br

** Graduada em Psicologia pela Universidade São Judas Tadeu. E-mail: daiana.albuquerque@ outlook.com

*** Graduada em Psicologia pela Universidade São Judas Tadeu. E-mail: fernandatqueiroz@ yahoo.com.br

**** Graduada em Psicologia pela Universidade São Judas Tadeu. E-mail: gabriela_plopes@ hotmail.com

***** Graduada em Psicologia pela Universidade São Judas Tadeu. E-mail: letviesilva@gmail.com
} 
O índice de evasão foi elevado (51,3\%), mas compatível com outras pesquisas da área. Em relação ao diagnóstico, as reações emocionais às relações familiares foram as problemáticas mais frequentes.

Palavras-chave: avaliação psicológica, clínica infantil, serviços-escola de psicologia.

\section{Abstract}

This study sought to characterize children, given the psychological diagnostic assessments from 2007 to 2013, at a therapy clinic with psychologists under training in the city of São Paulo. This quantitative and documental study was performed using surveys, book analyses, enrollment records, and reports of the children as observed at the clinic. Of all the children enrolled ( $n=150)$, 67.4\% were male and had been recommended by other healthcare professionals. Complaints about school were common among both boys and girls, as were the complaints of emotional reactions to family relationships. The drop-out rate was high (51.3\%) but compatible with other studies in this field. In terms of diagnoses, emotional reactions to family relationships were the most frequent issues.

Keywords: psychological assessment, children's clinics, services from psychologists in training

\section{Resumen}

El presente estudio objetivó caracterizar la clientela infantil atendida en evaluación psicodiagnóstica durante el periodo de 2007 a 2013, en una clínica- escuela de psicología, en la ciudad de São Paulo. Este estudio trata sobre una pesquisa documental, de naturaleza cuantitativa, realizada por medio de un levantamiento y análisis de libros, fichas de inscripción y prontuarios de los niños atendidos. Del total de niños inscritos ( $N=150)$, 67,4\% eran de sexo masculino, remetidos por profesionales de salud. Las quejas escolares fueron frecuentes en niños y niñas, justamente con quejas de reacciones emocionales a las relaciones emocionales. El índice de evasión fue elevado (51,3\%), pero compatible con otras investigaciones del área. En relación al diagnóstico, las reacciones emocionales y las relaciones familiares fueron las problemáticas más frecuentes.

Palabras clave: evaluación psicológica, clínica infantil, servicios-escuela de psicología

As clínicas-escola são obrigatórias na regulamentação dos cursos de graduação em Psicologia e fundamentam-se em três eixos básicos: o ensino, a extensão e a pesquisa. Esses centros de Psicologia Aplicada são essenciais 
para a consolidação da formação teórico-prática do futuro profissional e exercem uma função social relevante na prestação de serviços à comunidade (Boaz \& Nunes, 2010; Boaz, Nunes, \& Hirakata, 2012; Campezatto, Menegat, Nunes, \& Vitola, 2005; Campezatto \& Nunes, 2007; Macedo et al., 2011; Maravieski \& Serralta, 2011; Oliveira et al., 2014).

As clínicas-escola proporcionam o acesso a serviços psicológicos gratuitos ou de baixo custo, supervisionados por profissionais experientes e qualificados. Ressalta-se que grande parcela da população atendida nesses serviços não dispõe de recursos para arcar com os custos da assistência psicológica privada e que os serviços públicos de saúde mental são insuficientes para absorver a demanda (Amaral et al., 2012; Boaz et al., 2012; Borsa, Oliveira, Yates, \& Bandeira, 2013; Coelho, Peres, \& Oliveira, 2005).

A produção de pesquisas no contexto da clínica-escola permite conhecer "quem são, de onde vêm, o que buscam, como vivem e quais são as expectativas dos seus clientes" (Campezatto et al., 2005, p. 127) e reavaliar a efetividade das atividades de extensão. Estudos nessa área oportunizam a reflexão sobre a prática psicológica institucional e a revisão dos modelos adotados no atendimento de demandas da comunidade e no processo de ensino-aprendizagem (Melo-Silva, Santos \& Simon, 2005).

A literatura especializada mostra que a clientela infantil das clínicas-escola de Psicologia é predominantemente constituída por crianças do sexo masculino (Amaral et al., 2012; Boaz \& Nunes, 2010; Boaz et al., 2012; Rodrigues, Campos \& Fernandes, 2012; Borsa, Oliveira et al., 2013; Borsa, Segabinazi et al., 2013; Cunha \& Benetti, 2009; Lopes, Silva \& Vagostello, 2013; Mantovani, Marturano \& Silvares, 2010; Maravieski \& Serralta, 2011; Melo \& Perfeito, 2006; Merg, 2008; Moura, Marinho-Casanova, Meurer \& Campana, 2008; Scortegagna \& Levandowski, 2004; Wielewicki, 2011).

A extensa revisão de produção científica sobre demandas infantis nos serviços de clínicas-escola (Boaz \& Nunes, 2010) constatou que nos anos de 1980, a agressividade era a queixa mais frequente em meninos, a timidez mais comum em meninas e os problemas de aprendizagem recorrentes em ambos (Sales, 1989). Na década de 1990, as mesmas queixas permaneceram associadas ao sexo masculino, no entanto a incidência de queixas relacionadas à aprendizagem, agressividade e nervosismo aumentou entre 
as meninas (Barbosa \& Silvares, 1994; Borges, 1996; Graminha \& Martins, 1993). No último período (2000 a 2010), as demandas de agressividade mantiveram-se nos meninos e os sintomas do tipo internalizante (associados à depressão, ansiedade e somatização) destacaram-se nas meninas (Gatti \& Beres, 2004), porém com tendência à equiparação de queixas de agressividade entre ambos.

Na mesma vertente, o levantamento documental realizado por Boaz, Nunes e Hirakata (2012), em três clínicas-escola de Porto Alegre, encontrou o predomínio de queixas de ansiedade, depressão e retraimento associadas a meninos e queixas somáticas a meninas nos anos de 1980. Na década de 1990, as queixas relacionadas a comportamentos agressivos, atenção, ansiedade e depressão apareceram associadas a crianças do sexo masculino, porém não foram identificadas queixas significativamente associadas ao sexo feminino. Entre 2000 e 2010, o estudo identificou aproximação das queixas entre ambos os gêneros, com maior incidência de problemas de atenção em meninos e de retraimento e depressão em meninas. $\mathrm{O}$ levantamento em questão sugere que as queixas desenvolvimentais não se relacionam à variável gênero de maneira significativa (Boaz, Nunes \& Hirakata, 2012).

Confirmando a tendência verificada por Boaz e Nunes (2010), Borsa, Segabinazi et al. (2013) identificaram queixas de atenção significativamente associadas em crianças de ambos os sexos, sintomas do tipo externalizante (agressividade) associados a meninos e do tipo internalizante (ansiedade e depressão) a meninas. Resultados semelhantes também foram encontrados por Wielewicki (2011).

Cabe mencionar que queixas escolares relativas à dificuldade de aprendizagem são recorrentes entre a clientela infantil e figuram entre as três principais queixas atendidas nos centros de Psicologia aplicada dos cursos de graduação em Psicologia (Amaral et al., 2012; Borsa, Oliveira et al., 2013; Borsa, Segabinazi et al.,2013; Cunha \& Benetti, 2009; Rodrigues et al., 2012; Romaro \& Capitão, 2003).

A literatura especializada também revela que a evasão da clientela infantil das clínicas-escola tende a ser elevada e que cerca de 50\% das crianças inscritas abandonam o serviço antes ou após o início de alguma 
intervenção. Cunha e Benetti (2009) e Mantovani et al. (2010) encontraram índices ainda mais elevados de evasão, 64,5\% e 57\%, respectivamente. Nesse sentido, a compreensão mais apurada das características e demandas dos usuários de clínicas-escola favorece a oferta de serviços mais alinhados às necessidades dessa população, podendo ainda contribuir para a redução desses altos níveis de evasão (Amaral et al., 2012; Cunha \& Benetti, 2009; Santos, 1990, citado por Campezatto et al., 2005).

A partir dessa perspectiva, o presente estudo objetivou, por meio de uma pesquisa documental, caracterizar demandas de crianças encaminhadas para avaliação psicodiagnóstica em uma clínica-escola de Psicologia de São Paulo, no período de 2007 a 2013.

\section{MÉTODO}

A pesquisa documental ocorreu por meio do levantamento e da análise dos arquivos de uma clínica-escola de Psicologia em uma universidade privada localizada na região central da cidade de São Paulo. Após aprovação do projeto de pesquisa pelo Comitê de Ética (CAAE 30794814.8.0000.0089), iniciou-se a consulta das fichas de inscrição e dos prontuários de crianças de até 12 anos de idade incompletos, direcionadas para realização de avaliação psicodiagnóstica, no período de 2007 a 2013.

O levantamento das fichas de inscrição forneceu informações sobre o número, o sexo e a idade das crianças, as queixas fornecidas pelos responsáveis, as fontes de encaminhamento, os encaminhamentos realizados após o processo psicodiagnóstico e os índices de evasão.

A consulta dos prontuários possibilitou o acesso a informações mais detalhadas e precisas sobre as queixas e os diagnósticos realizados. De posse dessas informações foi possível identificar correspondências ou discrepâncias entre as queixas mencionadas pelos responsáveis e as problemáticas identificadas pelo processo psicodiagnóstico.

Os dados levantados foram organizados, agrupados e quantificados por meio de frequência e porcentagem. As queixas informadas pelos responsáveis das crianças foram classificadas em categorias, as mesmas 
empregadas por Romaro e Capitão (2003), porém com algumas alterações e reagrupamentos. As referidas categorias também foram utilizadas para classificar as problemáticas identificadas no processo psicodiagnóstico.

Foram utilizadas as seguintes categorias de classificação de queixas e diagnóstico: 1. Agressividade (dificuldade de controle da agressividade, comportamento destrutivo ou rebeldia); 2. Insegurança/Ansiedade (busca de atenção, manifestações de ansiedade, medo, roer unha, dentre outros); 3. Depressão/tristeza; 4. Dificuldade no controle dos impulsos (comportamento agitado, birra, choro excessivo, irritabilidade, oscilação de humor); 5. Dificuldade nos relacionamentos interpessoais (desobediência, teimosia, egoísmo, elevado nível de exigência, falsidade, timidez, problema na interação com colegas, isolamento social); 6. Distúrbios da alimentação (anorexia, bulimia, compulsão alimentar); 7. Imaturidade e atraso no desenvolvimento (dificuldades motoras, de aprendizagem, de hábitos rotineiros, de discriminações básicas; lentidão); 8. Problemas de conduta (fugas de casa ou da escola, comportamento manipulador, mentira, roubo, adição a drogas); 9. Problemas neurológicos (disfunções neurológicas, lesões neurológicas); 10. Queixas escolares (desempenho abaixo do esperado, comportamento em desacordo com as normas escolares, resistência à escola); 11. Queixas somatoformes (desmaios, cefaleias, bronquite, dores no estômago, vômitos, dores em geral); 12. Reações emocionais às relações familiares (ciúmes, medo dos pais, dificuldade em relação aos pais e familiares; dificuldade com a separação dos pais, ligação excessiva com a família, sentimento de rejeição, reação diante da perda por morte ou por separação).

Algumas queixas que ocorreram com frequência reduzida foram classificadas como "Outras queixas".

\section{RESULTADOS}

No período de sete anos (2007-2013) a clínica-escola registrou 150 crianças inscritas para avaliação psicodiagnóstica, 101 do sexo masculino e 49 do sexo feminino. $\mathrm{O}$ ano de 2007 teve o maior número de crianças $(\mathrm{N}=40)$, com expressiva queda nos anos subsequentes, conforme mostra a tabela 1: 
Tabela 1 - Crianças inscritas para avaliação psicodiagnóstica

\begin{tabular}{|c|c|c|c|c|c|c|c|c|c|c|c|c|c|c|c|c|}
\hline \multirow{2}{*}{ Sexo } & \multicolumn{2}{|c|}{2007} & \multicolumn{2}{|c|}{2008} & \multicolumn{2}{|c|}{2009} & \multicolumn{2}{|c|}{2010} & \multicolumn{2}{|c|}{2011} & \multicolumn{2}{|c|}{2012} & \multicolumn{2}{|c|}{2013} & \multicolumn{2}{|c|}{ Total } \\
\hline & f & $\%$ & f & $\%$ & f & $\%$ & f & $\%$ & f & $\%$ & f & $\%$ & f & $\%$ & f & $\%$ \\
\hline Masculino & 22 & 55,0 & 10 & 71,4 & 11 & 64,7 & 19 & 76,0 & 11 & 68,8 & 10 & 71,4 & 18 & 75,0 & 101 & 67,4 \\
\hline Feminino & 18 & 45,0 & 4 & 28,6 & 6 & 35,3 & 6 & 24,0 & 5 & 31,2 & 4 & 28,6 & 6 & 25,0 & 49 & 32,6 \\
\hline Total por ano & 40 & 100,0 & 14 & 100,0 & 17 & 100,0 & 25 & 100,0 & 16 & 100,0 & 14 & 100,0 & 24 & 100,0 & 150 & 100,0 \\
\hline
\end{tabular}

No decorrer desse período, a proporção de meninos atendidos $(67,4 \%)$ foi muito superior ao de meninas $(32,6 \%)$, mais do que o dobro. No ano de 2007 a diferença foi mais equilibrada (55\% e $45 \%$, respectivamente) e nos anos de 2010 e de 2013 o número de meninos atendidos foi três vezes superior ao de meninas.

A maior parte das crianças inscritas concentra-se nas faixas etárias de 9 a 10 anos $(36,7 \%)$ e de 7 a 8 anos de idade $(26,7 \%)$. A Tabela 2 apresenta a distribuição das crianças, por faixa etária, no período de sete anos:

Tabela 2 - Crianças inscritas para psicodiagnóstico por faixa etária

\begin{tabular}{|c|c|c|c|c|c|c|c|c|c|c|c|c|c|c|c|c|}
\hline \multirow{2}{*}{ Faixa Etária } & \multicolumn{2}{|c|}{2007} & \multicolumn{2}{|c|}{2008} & \multicolumn{2}{|c|}{2009} & \multicolumn{2}{|c|}{2010} & \multicolumn{2}{|c|}{2011} & \multicolumn{2}{|c|}{2012} & \multicolumn{2}{|c|}{2013} & \multicolumn{2}{|c|}{ Total } \\
\hline & $f$ & $\%$ & $\mathrm{f}$ & $\%$ & f & $\%$ & $f$ & $\%$ & $f$ & $\%$ & $f$ & $\%$ & $f$ & $\%$ & $\mathrm{f}$ & $\%$ \\
\hline 3 a 4 anos & 1 & 2,5 & o & 0,0 & o & 0,0 & o & 0,0 & 1 & 6,2 & 0 & 0,0 & 1 & 4,2 & 3 & 2,0 \\
\hline 5 a 6 anos & 8 & 20,0 & 4 & 28,6 & 4 & 23,5 & 5 & 20,0 & 3 & 18,8 & 3 & 21,4 & 5 & 20,8 & 32 & 21,3 \\
\hline 7 a 8 anos & 12 & 30,0 & 4 & 28,6 & 4 & 23,5 & 7 & 28,0 & 4 & 25,0 & 3 & 21,4 & 6 & 25,0 & 40 & 26,7 \\
\hline 9 a 10 anos & 12 & 30,0 & 6 & 42,8 & 6 & 35,3 & 10 & 40,0 & 7 & 43,8 & 6 & 42,8 & 8 & 33,3 & 55 & 36,7 \\
\hline $\begin{array}{l}11 \text { a } 12 \text { anos } \\
\text { incompletos }\end{array}$ & 7 & 17,5 & 0 & 0,0 & 3 & 17,7 & 3 & 12,0 & 1 & 6,2 & 2 & 14,4 & 4 & 16,7 & 20 & 13,3 \\
\hline Total por ano & 40 & 100,0 & 14 & 100,0 & 17 & 100,0 & 25 & 100,0 & 16 & 100,0 & 14 & 100,0 & 24 & 100,0 & 150 & 100,0 \\
\hline
\end{tabular}

Constatou-se que o acesso aos serviços da clínica-escola ocorreu por indicação de profissionais da saúde (24,7\%), de amigos (22,0\%) e de escolas ou instituições de abrigo (20,0\%). Outras fontes mencionadas foram alunos e funcionários da universidade, programas de inclusão social (11,4\%), família (8,0\%) e mídia (6,6\%). Em 7,3\% dos casos, as fontes de encaminhamento não foram informadas.

As queixas escolares (27,0\%) foram as mais frequentes em crianças do sexo masculino ( $\mathrm{N}=101)$, seguidas por agressividade $(18,8 \%)$, dificuldade no controle dos impulsos $(18,8 \%)$ e reações emocionais às relações familiares 
(13,9\%). É importante esclarecer que muitas crianças apresentaram mais de uma queixa, razão pela qual o número de queixas é superior ao número de crianças inscritas, conforme mostra a tabela 3, a seguir.

Tabela 3 - Queixas em meninos no período de 2007 a 2013

\begin{tabular}{|c|c|c|c|c|c|c|c|c|c|c|c|c|c|c|c|c|}
\hline \multirow{2}{*}{ Queixas } & \multicolumn{2}{|c|}{2007} & \multicolumn{2}{|c|}{2008} & \multicolumn{2}{|c|}{2009} & \multicolumn{2}{|c|}{2010} & \multicolumn{2}{|c|}{2011} & \multicolumn{2}{|c|}{2012} & \multicolumn{2}{|c|}{2013} & \multicolumn{2}{|c|}{ Total } \\
\hline & f & $\%$ & f & $\%$ & f & $\%$ & f & $\%$ & f & $\%$ & f & $\%$ & f & $\%$ & f & $\%$ \\
\hline Queixas escolares & 9 & 23,1 & 3 & 21,4 & 2 & 12,5 & 6 & 26,1 & 5 & 29,4 & 4 & 66,6 & 10 & 34,5 & 39 & 27,0 \\
\hline Agressividade & 7 & 17,9 & 2 & 14,4 & 4 & 25,0 & 6 & 26,1 & 4 & 23,6 & 0 & 0,0 & 4 & 13,8 & 27 & 18,8 \\
\hline $\begin{array}{l}\text { Dificuldade no } \\
\text { controle de } \\
\text { impulsos }\end{array}$ & 8 & 20,5 & 3 & 21,4 & 2 & 12,5 & 2 & 8,7 & 2 & 11,8 & 0 & 0,0 & 10 & 34,5 & 27 & 18,8 \\
\hline $\begin{array}{c}\text { Reações } \\
\text { emocionais às } \\
\text { relações familiares }\end{array}$ & 5 & 12,8 & 1 & 7,1 & 1 & 6,3 & 7 & 30,4 & 3 & 17,6 & 1 & 16,7 & 2 & 6,9 & 20 & 13,9 \\
\hline $\begin{array}{l}\text { Ansiedade / } \\
\text { Insegurança }\end{array}$ & 3 & 7,7 & 0 & 0,0 & 2 & 12,5 & 0 & 0,0 & 0 & 0,0 & 0 & 0,0 & 2 & 6,9 & 7 & 4,8 \\
\hline $\begin{array}{l}\text { Dificuldades nos } \\
\text { relacionamentos } \\
\text { interpessoais }\end{array}$ & 2 & 5,1 & 0 & 0,0 & 2 & 12,5 & 0 & 0,0 & 3 & 17,6 & 0 & 0,0 & 0 & 0,0 & 7 & 4,8 \\
\hline $\begin{array}{c}\text { Queixas } \\
\text { somatoformes }\end{array}$ & 3 & 7,7 & 1 & 7,1 & 0 & 0,0 & 0 & 0,0 & 0 & 0,0 & 0 & 0,0 & 0 & 0,0 & 4 & 2,8 \\
\hline Outras queixas & 0 & 0,0 & 1 & 7,1 & 0 & 0,0 & 2 & 8,7 & 0 & 0,0 & 0 & 0,0 & 1 & 3,4 & 4 & 2,8 \\
\hline $\begin{array}{c}\text { Imaturidade } \\
\text { e atraso no } \\
\text { desenvolvimento }\end{array}$ & 0 & 0,0 & 2 & 14,4 & 1 & 6,3 & o & 0,0 & 0 & 0,0 & 0 & 0,0 & o & 0,0 & 3 & 2,1 \\
\hline $\begin{array}{c}\text { Depressão / } \\
\text { Tristeza }\end{array}$ & 1 & 2,6 & 0 & 0,0 & 1 & 6,3 & 0 & 0,0 & 0 & 0,0 & 0 & 0,0 & 0 & 0,0 & 2 & 1,4 \\
\hline $\begin{array}{c}\text { Problemas } \\
\text { neurológicos }\end{array}$ & 1 & 2,6 & 0 & 0,0 & 1 & 6,3 & 0 & 0,0 & 0 & 0,0 & 0 & 0,0 & 0 & 0,0 & 2 & 1,4 \\
\hline $\begin{array}{l}\text { Problemas de } \\
\text { conduta }\end{array}$ & 0 & 0,0 & 1 & 7,1 & 0 & 0,0 & 0 & 0,0 & 0 & 0,0 & 1 & 16,7 & 0 & 0,0 & 2 & 1,4 \\
\hline Total por ano & 39 & 100,0 & 14 & 100,0 & 16 & 100,0 & 23 & 100,0 & 17 & 100,0 & 6 & 100,0 & 29 & 100,0 & 144 & 100,0 \\
\hline
\end{tabular}

As queixas escolares $(17,6 \%)$ também foram as mais frequentes no grupo feminino $(\mathrm{N}=49)$, juntamente com as reações emocionais às relações familiares (17,6\%). A dificuldade no controle dos impulsos (13,5\%) e a agressividade (12,2\%) também se destacaram neste grupo, porém em menor proporção do que em meninos, segundo demonstra a tabela 4 . 
Tabela 4 - Queixas em meninas no período de 2007 a 2013

\begin{tabular}{|c|c|c|c|c|c|c|c|c|c|c|c|c|c|c|c|c|}
\hline \multirow{2}{*}{ Queixas } & \multicolumn{2}{|c|}{2007} & \multicolumn{2}{|c|}{2008} & \multicolumn{2}{|c|}{2009} & \multicolumn{2}{|c|}{2010} & \multicolumn{2}{|c|}{2011} & \multicolumn{2}{|c|}{2012} & \multicolumn{2}{|c|}{2013} & \multicolumn{2}{|c|}{ Total } \\
\hline & $\mathbf{f}$ & $\%$ & $\mathbf{f}$ & $\%$ & $\mathbf{f}$ & $\%$ & f & $\%$ & $\mathbf{f}$ & $\%$ & $\mathbf{f}$ & $\%$ & f & $\%$ & f & $\%$ \\
\hline Queixas escolares & 4 & 15,5 & 1 & 14,3 & 3 & 30,0 & 1 & 20,0 & 1 & 20,0 & 1 & 10,0 & 2 & 18,2 & 13 & 17,6 \\
\hline $\begin{array}{c}\text { Reações } \\
\text { emocionais às } \\
\text { relações familiares }\end{array}$ & 6 & 23,1 & 2 & 28,5 & 0 & 0,0 & 0 & 0,0 & 1 & 20,0 & 3 & 30,0 & 1 & 9,1 & 13 & 17,6 \\
\hline $\begin{array}{l}\text { Ansiedade / } \\
\text { Insegurança }\end{array}$ & 3 & 11,5 & 0 & 0,0 & 3 & 30,0 & 0 & 0,0 & 1 & 20,0 & 3 & 30,0 & 1 & 9,1 & 11 & 14,9 \\
\hline $\begin{array}{c}\text { Dificuldade no } \\
\text { controle de } \\
\text { impulsos }\end{array}$ & 3 & 11,5 & 1 & 14,3 & 2 & 20,0 & 1 & 20,0 & 0 & 0,0 & 1 & 10,0 & 2 & 18,2 & 10 & 13,5 \\
\hline Agressividade & 3 & 11,5 & 1 & 14,3 & 1 & 10,0 & 1 & 20,0 & 0 & 0,0 & 1 & 10,0 & 2 & 18,2 & 9 & 12,2 \\
\hline $\begin{array}{c}\text { Queixas } \\
\text { somatoformes }\end{array}$ & 4 & 15,5 & 0 & 0,0 & 0 & 0,0 & 1 & 20,0 & 1 & 20,0 & 0 & 0,0 & 1 & 9,1 & 7 & 9,5 \\
\hline $\begin{array}{l}\text { Dificuldades nos } \\
\text { relacionamentos } \\
\text { interpessoais }\end{array}$ & 1 & 3,8 & 1 & 14,3 & 1 & 10,0 & 1 & 20,0 & 1 & 20,0 & 0 & 0,0 & 0 & 0,0 & 5 & 6,7 \\
\hline $\begin{array}{c}\text { Imaturidade } \\
\text { e atraso no } \\
\text { desenvolvimento }\end{array}$ & 1 & 3,8 & 1 & 14,3 & 0 & 0,0 & 0 & 0,0 & 0 & 0,0 & 1 & 10,0 & 0 & 0,0 & 3 & 4,1 \\
\hline $\begin{array}{l}\text { Distúrbios da } \\
\text { alimentação }\end{array}$ & 1 & 3,8 & o & 0,0 & 0 & 0,0 & 0 & 0,0 & 0 & 0,0 & 0 & 0,0 & 1 & 9,1 & 2 & 2,6 \\
\hline Outras queixas & 0 & 0,0 & 0 & 0,0 & 0 & 0,0 & 0 & 0,0 & 0 & 0,0 & 0 & 0,0 & 1 & 9,1 & 1 & 1,3 \\
\hline Total por ano & 26 & 100,0 & 7 & 100,0 & 10 & 100,0 & 5 & 100,0 & 5 & 100,0 & 10 & 100,0 & 11 & 100,0 & 74 & 100,0 \\
\hline
\end{tabular}

As queixas escolares foram as mais frequentes tanto no grupo masculino, como no feminino. Os dois grupos também apresentaram outras quatro categorias de queixas mais recorrentes (agressividade, dificuldade no controle dos impulsos, ansiedade/insegurança e reações emocionais às relações familiares), porém em proporções distintas.

No que se refere às problemáticas identificadas no processo psicodiagnóstico, as reações emocionais às relações familiares $(37,1 \%)$ foi a mais frequente, seguida pela imaturidade/atraso no desenvolvimento $(15,9 \%)$ e agressividade (12,1\%).

É importante destacar que não foram identificadas problemáticas complexas ou de transtornos psicológicos em 7,5\% das crianças submetidas ao processo psicodiagnóstico. Esse resultado é a quarta categoria mais frequente no grupo total de crianças (meninas e meninos), conforme mostra a tabela 5 . 


\section{Tabela 5 - Problemáticas identificadas no processo} psicodiagnóstico no grupo total de crianças

\begin{tabular}{|c|c|c|c|c|c|c|c|c|c|c|c|c|c|c|c|c|}
\hline \multirow{2}{*}{ Psicodiagnóstico } & \multicolumn{2}{|c|}{2007} & \multicolumn{2}{|c|}{2008} & \multicolumn{2}{|c|}{2009} & \multicolumn{2}{|c|}{2010} & \multicolumn{2}{|c|}{2011} & \multicolumn{2}{|c|}{2012} & \multicolumn{2}{|c|}{2013} & \multicolumn{2}{|c|}{ Total } \\
\hline & f & $\%$ & f & $\%$ & $\mathbf{f}$ & $\%$ & $\mathbf{f}$ & $\%$ & f & $\%$ & f & $\%$ & f & $\%$ & f & $\%$ \\
\hline $\begin{array}{c}\text { Reações emocionais } \\
\text { às relações } \\
\text { familiares }\end{array}$ & 16 & 36,4 & 3 & 37,5 & 5 & 55,6 & 7 & 31,8 & 4 & 36,4 & 4 & 44,5 & 10 & 34,5 & 49 & 37,1 \\
\hline $\begin{array}{c}\text { Imaturidade } \\
\text { e atraso no } \\
\text { desenvolvimento }\end{array}$ & 9 & 20,5 & 4 & 50,0 & 1 & 11,1 & 2 & 9,1 & 2 & 18,2 & 1 & 11,1 & 2 & 6,9 & 21 & 15,9 \\
\hline Agressividade & 5 & 11,4 & 0 & 0,0 & 0 & 0,0 & 3 & 13,7 & 0 & 0,0 & 1 & 11,1 & 7 & 24,1 & 16 & 12,1 \\
\hline $\begin{array}{l}\text { Crianças com } \\
\text { ausência de } \\
\text { problemática } \\
\text { complexa ou } \\
\text { transtorno }\end{array}$ & 2 & 4,5 & 0 & 0,0 & 0 & 0,0 & 4 & 18,2 & 3 & 27,2 & 1 & 11,1 & 0 & 0,0 & 10 & 7,5 \\
\hline $\begin{array}{l}\text { Ansiedade / } \\
\text { Insegurança }\end{array}$ & 1 & 2,3 & 1 & 12,5 & 0 & 0,0 & 1 & 4,5 & 1 & 9,1 & 1 & 11,1 & 2 & 6,9 & 7 & 5,3 \\
\hline $\begin{array}{c}\text { Dificuldades } \\
\text { escolares }\end{array}$ & 0 & 0,0 & 0 & 0,0 & 0 & 0,0 & 3 & 13,7 & 1 & 9,1 & 1 & 11,1 & 2 & 6,9 & 7 & 5,3 \\
\hline $\begin{array}{l}\text { Dificuldade no } \\
\text { controle de } \\
\text { impulsos }\end{array}$ & 6 & 13,5 & 0 & 0,0 & 0 & 0,0 & 0 & 0,0 & 0 & 0,0 & 0 & 0,0 & 0 & 0,0 & 6 & 4,5 \\
\hline $\begin{array}{c}\text { Depressão / } \\
\text { Tristeza }\end{array}$ & 1 & 2,3 & 0 & 0,0 & 2 & 22,2 & 0 & 0,0 & 0 & 0,0 & 0 & 0,0 & 2 & 6,9 & 5 & 3,8 \\
\hline $\begin{array}{l}\text { Dificuldades nos } \\
\text { relacionamentos } \\
\text { interpessoais }\end{array}$ & 1 & 2,3 & 0 & 0,0 & 0 & 0,0 & 0 & 0,0 & 0 & 0,0 & 0 & 0,0 & 3 & 10,3 & 4 & 3,1 \\
\hline $\begin{array}{l}\text { Problemas } \\
\text { neurológicos }\end{array}$ & 2 & 4,5 & 0 & 0,0 & 0 & 0,0 & 0 & 0,0 & 0 & 0,0 & 0 & 0,0 & 0 & 0,0 & 2 & 1,5 \\
\hline $\begin{array}{c}\text { Queixas } \\
\text { somatoformes }\end{array}$ & 0 & 0,0 & 0 & 0,0 & 0 & 0,0 & 1 & 4,5 & 0 & 0,0 & 0 & 0,0 & 1 & 3,5 & 2 & 1,5 \\
\hline $\begin{array}{l}\text { Distúrbios de } \\
\text { alimentação }\end{array}$ & 1 & 2,3 & 0 & 0,0 & 0 & 0,0 & 0 & 0,0 & 0 & 0,0 & 0 & 0,0 & 0 & 0,0 & 1 & 0,8 \\
\hline $\begin{array}{c}\text { Conflito de } \\
\text { identidade sexual }\end{array}$ & 0 & 0,0 & 0 & 0,0 & 1 & 11,1 & 0 & 0,0 & 0 & 0,0 & 0 & 0,0 & 0 & 0,0 & 1 & 0,8 \\
\hline Trantorno psicótico & 0 & 0,0 & 0 & 0,0 & 0 & 0,0 & 1 & 4,5 & 0 & 0,0 & 0 & 0,0 & 0 & 0,0 & 1 & 0,8 \\
\hline Total por ano & 44 & 100,0 & 8 & 100,0 & 9 & 100,0 & 22 & 100,0 & 11 & 100,0 & 9 & 100,0 & 29 & 100,0 & 132 & 100,0 \\
\hline
\end{tabular}

Analisando separadamente meninos e meninas, constatou-se que as reações emocionais às relações familiares e a imaturidade/atraso no desenvolvimento foram as problemáticas mais recorrentes tanto em crianças do sexo feminino ( $42,2 \%$ e $13,3 \%$, respectivamente) como do sexo masculino (34,5\% e 17,2\%, respectivamente). A terceira categoria mais frequente em meninos foi a agressividade $(13,8 \%)$ e em meninas foi a dificuldade no controle dos impulsos (11,1\%).

Após a conclusão do processo psicodiagnóstico, 46,9\% das crianças foram encaminhadas para psicoterapia e 29,2\% dos familiares (pais) foram 
encaminhados para intervenções individuais ou grupais (psicoterapia familiar, grupo de pais) na própria clínica-escola. Em 11,5\% dos casos, as crianças receberam alta, conforme ilustra a tabela a seguir (tabela 6).

Tabela 6 - Encaminhamentos para crianças e familiares

\begin{tabular}{|c|c|c|c|c|c|c|c|c|c|c|c|c|c|c|c|c|}
\hline \multirow{2}{*}{$\begin{array}{l}\text { Encaminhamentos } \\
\text { Realizados }\end{array}$} & \multicolumn{2}{|c|}{2007} & \multicolumn{2}{|c|}{2008} & \multicolumn{2}{|c|}{2009} & \multicolumn{2}{|c|}{2010} & \multicolumn{2}{|c|}{2011} & \multicolumn{2}{|c|}{2012} & \multicolumn{2}{|c|}{2013} & \multicolumn{2}{|c|}{ Total } \\
\hline & f & $\%$ & f & $\%$ & f & $\%$ & f & $\%$ & f & $\%$ & f & $\%$ & f & $\%$ & f & $\%$ \\
\hline Interno para crianças & 12 & 54,5 & 2 & 40,0 & 3 & 50,0 & 10 & 40,0 & 5 & 38,5 & 5 & 41,7 & 8 & 61,5 & 45 & 46,9 \\
\hline Interno para famílias & 1 & 4,5 & 2 & 40,0 & 2 & 33,3 & 10 & 40,0 & 5 & 38,5 & 5 & 41,7 & 3 & 23,1 & 28 & 29,2 \\
\hline Alta para crianças & 2 & 9,1 & 0 & 0,0 & 0 & 0,0 & 4 & 16,0 & 2 & 15,4 & 2 & 16,6 & 1 & 7,7 & 11 & 11,5 \\
\hline Externo para crianças & 5 & 22,8 & 1 & 20,0 & 1 & 16,7 & 0 & 0,0 & 1 & 7,6 & 0 & 0,0 & 1 & 7,7 & 9 & 9,3 \\
\hline Externo para famílias & 2 & 9,1 & 0 & 0,0 & 0 & 0,0 & 1 & 4,0 & 0 & 0,0 & 0 & 0,0 & o & 0,0 & 3 & 3,1 \\
\hline Total por ano & 22 & 100,0 & 5 & 100,0 & 6 & 100,0 & 25 & 100,0 & 13 & 100,0 & 12 & 100,0 & 13 & 100,0 & 96 & 100,0 \\
\hline
\end{tabular}

Do total de crianças inscritas ( $\mathrm{N}=150)$, apenas $48,7 \%$ encerraram o processo psicodiagnóstico ( $\mathrm{N}=73$ ), ou seja, o índice de evasão foi de $51,3 \%$. Do total de evasões, 32,7\% ocorreram antes do início do processo e 18,6\% durante o processo, conforme indica a Tabela 7.

Tabela 7 - Distribuição de adesão ao atendimento e índice de evasão

\begin{tabular}{|c|c|c|c|c|c|c|c|c|c|c|c|c|c|c|c|c|}
\hline \multirow{2}{*}{$\begin{array}{c}\text { Adesão / } \\
\text { evasão do } \\
\text { atendimento }\end{array}$} & \multicolumn{2}{|c|}{2007} & \multicolumn{2}{|c|}{2008} & \multicolumn{2}{|c|}{2009} & \multicolumn{2}{|c|}{2010} & \multicolumn{2}{|c|}{2011} & \multicolumn{2}{|c|}{2012} & \multicolumn{2}{|c|}{2013} & \multicolumn{2}{|c|}{ Total } \\
\hline & $\mathbf{f}$ & $\%$ & $\mathbf{f}$ & $\%$ & $\mathbf{f}$ & $\%$ & $\mathbf{f}$ & $\%$ & $\mathbf{f}$ & $\%$ & $\mathbf{f}$ & $\%$ & $\mathbf{f}$ & $\%$ & f & $\%$ \\
\hline $\begin{array}{c}\text { Adesão ao } \\
\text { atendimento }\end{array}$ & 21 & 52,5 & 5 & 35,7 & 6 & 35,3 & 14 & 56,0 & 8 & 50,0 & 7 & 50,0 & 12 & 50,0 & 73 & 48,7 \\
\hline $\begin{array}{l}\text { Usuário nunca } \\
\text { apareceu } \\
\text { (evasão antes } \\
\text { atendimento) }\end{array}$ & 16 & 40,0 & 7 & 50,0 & 4 & 23,5 & 3 & 12,0 & 5 & 31,2 & 5 & 35,7 & 9 & 37,5 & 49 & 32,7 \\
\hline $\begin{array}{l}\text { Evasão durante } \\
\text { atendimento } \\
\text { psicodiagnóstico }\end{array}$ & 3 & 7,5 & 2 & 14,3 & 7 & 41,2 & 8 & 32,0 & 3 & 18,8 & 2 & 14,3 & 3 & 12,5 & 28 & 18,6 \\
\hline Total por ano & 40 & 100,0 & 14 & 100,0 & 17 & 100,0 & 25 & 100,0 & 16 & 100,0 & 14 & 100,0 & 24 & 100,0 & 150 & 100,0 \\
\hline
\end{tabular}

\section{DISCUSSÃO}

Sabe-se que a procura por atendimentos psicológicos em clínicas-escola tende a ser elevada uma vez que essa prestação de serviços em clínicas privadas é pouco acessível para grande parte da população (Campezatto \& Nunes, 2007). No entanto, foi constatada expressiva redução no 
número de crianças direcionadas anualmente para avaliação psicodiagnóstica após 2007, o que pode ser atribuído a mudanças nos procedimentos de triagem ocorridas naquele período.

O número de crianças do sexo masculino inscritas na clínica-escola é duas vezes maior do que o de meninas, resultado que converge com a literatura (Boaz \& Nunes, 2010; Boaz et al., 2012; Borsa, Oliveira et al., 2013; Borsa, Segabinazi et al., 2013; Campezatto \& Nunes, 2007; Cunha \& Benetti, 2009; Gatti \& Beres, 2004; Melo \& Perfeito, 2006; Merg, 2008; Santa-Rosa, 2008). Alguns autores relacionam esse fenômeno à incidência de comportamentos externalizantes em meninos, cuja reprovação social é maior, sobretudo nos contextos escolar e familiar (Campezatto \& Nunes, 2007; Merg, 2008; Wielewicki, 2011).

A faixa etária prevalente foi de 9 e 10 anos, resultado que coincide com os primeiros anos do ensino formal, período em que a criança migra do ambiente familiar para um contexto social mais abrangente e exigente. A integração da criança à realidade escolar requer mobilização de recursos adaptativos, sociais e cognitivos para conquistar novas aprendizagens e tolerar frustrações. Não por acaso que a categoria mais frequente de queixas foi a escolar, confirmando a tendência apresentada em estudos da área (Boaz \& Nunes, 2010; Cunha \& Benetti, 2009; Gatti \& Beres, 2004; Merg, 2008; Rodrigues et al., 2012; Romaro \& Capitão, 2003; Scortegagna \& Levandowski, 2004).

Apesar de as queixas escolares terem sido mencionadas com elevada incidência e as instituições de ensino serem importantes fontes dos encaminhamentos psicológicos (Campezatto \& Nunes, 2007; Cunha e Benetti, 2009; Rodrigues et al., 2012), no presente estudo o encaminhamento das crianças ocorreu predominantemente por profissionais da saúde, resultado também encontrado na literatura (Borsa, Oliveira et al., 2013; Melo \& Perfeito, 2006). A progressiva valorização da interdisciplinaridade na área da saúde tende a estimular o encaminhamento por diferentes profissionais. Além disso, os temas psicológicos tornaram-se mais difundidos pelas mais variadas mídias sociais, o que pode explicar também a representativa incidência de encaminhamentos realizados pelo público leigo (amigos). 
Neste estudo, as cinco categorias mais frequentes de queixas foram as mesmas para meninos e meninas, com variações em relação à proporção de cada uma delas. Esse resultado é compatível com a tendência encontrada em estudos mais recentes que constataram maior aproximação nas queixas de crianças de gêneros diferentes (Boaz \& Nunes, 2010; Boaz et al., 2012). A agressividade, por exemplo, citada na literatura como uma das principais queixas infantis (Campezatto \& Nunes, 2007; Cunha \& Benetti, 2009; Gatti \& Beres, 2004; Melo \& Perfeito, 2006; Romaro \& Capitão, 2003), foi a segunda queixa mais frequente em meninos, mas também apareceu em meninas, porém em menor frequência do que os "comportamentos internalizantes".

A despeito da elevada incidência de queixas escolares, as problemáticas identificadas no processo diagnóstico como reações emocionais às relações familiares, observadas na maior parte das crianças do presente estudo, remetem-se a conflitos familiares e/ou de suporte ambiental (Cunha \& Benetti, 2009; Melo \& Perfeito, 2006).

Todavia, as questões relativas à imaturidade e ao atraso no desenvolvimento configuraram como a segunda categoria mais frequente no presente estudo, indicando a necessidade de intervenções interdisciplinares e articuladas com diversos saberes, para a compreensão mais abrangente do caso e efetividade do tratamento. Ressalta-se ainda que a escassez de profissionais especializados na rede pública de atendimento (psicopedagogos, fonoaudiólogos, neuropsicólogos, neurologistas, fisioterapeutas, psiquiatras infantis), dificulta o encaminhamento e a adesão ao tratamento após o processo psicodiagnóstico.

A ausência de conflitos emocionais significativos ou de transtornos associados à criança foi a quarta categoria psicodiagnóstica mais frequente na clínica-escola estudada. Muitas vezes o principal foco da intervenção não é a criança, mas a família, que correspondeu ao segundo maior alvo dos encaminhamentos da clínica-escola no presente estudo.

A evasão de mais da metade dos inscritos representa um índice elevado, porém convergente com a literatura pesquisada (Amaral et al., 2012; Cunha \& Benetti, 2009; Mantovani et al., 2010; Romaro \& Capitão, 2003). A atenuação do sintoma da criança pode ocorrer, em alguma medida, 
durante o processo psicodiagnóstico e, com isso, outros conflitos da dinâmica familiar tendem a ser explicitados. Quando os pais não conseguem entrar em contato com esses conflitos, a melhora no sintoma dos filhos pode se tornar ameaçadora e contribuir significativamente para a evasão. Não é por acaso que, segundo Cunha e Benetti (2009), o público infantil é o que mais procura, mais recebe e, também, é o que mais abandona os serviços de atendimentos psicoterapêuticos.

\section{CONSIDERAÇÕES FINAIS}

O levantamento das queixas iniciais e dos respectivos psicodiagnósticos permitiu mapear, com maior precisão, as demandas infantis da instituição estudada. A disparidade entre queixas e diagnósticos ressalta a importância do processo psicodiagnóstico, uma vez que as crianças e suas famílias podem, a partir dele, ser encaminhadas para modalidades psicoterapêuticas ajustadas às suas reais necessidades.

A identificação de diagnósticos relacionados à imaturidade cognitiva e ao atraso no desenvolvimento requer a constante reflexão sobre a interdisciplinaridade na formação de psicólogos e sobre a necessária articulação das clínicas-escola com a rede pública de atendimento. Por outro lado, a efetividade dos encaminhamentos de crianças com demandas neurológicas é limitada, uma vez que requer a intervenção de serviços especializados, geralmente indisponíveis na região dos usuários da clínica-escola.

A disfunção familiar muitas vezes é explicitada pela criança, mas nem sempre ela é o foco principal da intervenção. O elevado índice de evasão da clientela infantil evidencia a necessidade de colocar a família no centro das atenções da clínica-escola. A reavaliação dos modelos tradicionais de atendimento familiar e a oferta de modalidades de intervenção diversificadas para as famílias podem colaborar, de maneira significativa, com a redução da evasão da população infantil das clínicas-escola de Psicologia. 


\section{REFERÊNCIAS}

Amaral, A. E. V., Luca, L., Rodrigues, T. C., Leite, C. A., Lopes, F. L., \& Silva, M. A. (2012). Serviços de psicologia em clínicas-escola: revisão de literatura. Boletim de Psicologia, 62(136), 37-52. Recuperado de http://pepsic.bvsalud.org/scielo.php?script=sci_ arttext\&pid=So006-59432012000100005\&lng=pt\&tln $\mathrm{g}=\mathrm{pt}$

Barbosa, J. I. C., \& Silvares, E. F. M. (1994). Uma caracterização preliminar das clínicas-escola de Fortaleza. Estudos de Psicologia, 11(3), 50-56.

Boaz, C., \& Nunes, M. L. T. (2010, setembro/dezembro). Revisão da literatura brasileira sobre a problemática do desenvolvimento de crianças assistidas por clínicas-escola. Aletheia, (33), 151-165. Recuperado de http://pepsic.bvsalud.org/scielo.php?script=sci_ arttext\&pid=S1413-03942010000300013\&lng =pt\&tln $\mathrm{g}=\mathrm{pt}$

Boaz, C., Nunes, M. L. T., \& Hirakata, V. N. (2012, julho/setembro). A problemática do desenvolvimento de crianças assistidas por clínicasescola brasileiras mudaram no decorrer das décadas? Psico, 43(3), 334-340. Recuperado de http://revistaseletronicas.pucrs.br/ojs/ index.php/revistapsico/article/view/8328

Borges, S. L. P. (1996). Caracterização da clientela da Clínica São Marcos na área de atendimento infantil. Interações: Estudos e Pesquisa em Psicologia, 1(1), 59-78.

Borsa, J. C., Oliveira, S. E. S., Yates, D. B., \& Bandeira, D. R. (2013). Centro de Avaliação Psicológica - CAP: uma clínica-escola especializada em avaliação e diagnóstico psicológico. Psicologia Clínica, 25(1), 101-114. doi: 10.1590/So103-56652013000100007

Borsa, J. C., Segabinazi, J. D., Stenert, F., Yates, D. B., \& Bandeira, D. R. (2013, janeiro/março). Caracterização da clientela infantojuvenil de uma clínica-escola de avaliação psicológica de uma universidade brasileira. Psico, 44(1), 73-81. Recuperado de http:// revistaseletronicas.pucrs.br/ojs/index.php/revistapsico/article/ view/10599 
Campezatto, P. V. M., Menegat, C. B., Nunes, M. L. T., \& Vitola, J. C. (2005). Interface entre a Psicologia Clínica e a Psicologia da Saúde no Serviço de Atendimento Psicológico da PUC-RS. In L. L. Melo-Silva, M. A. Santos \& C. P. Simon (Orgs.), Formação em psicologia: serviçosescola em debate (pp. 119-137). São Paulo, SP: Vetor.

Campezatto, P. V. M., \& Nunes, M. L. T. (2007). Caracterização da clientela das clínicas-escola de cursos de Psicologia da região metropolitana de Porto Alegre. Psicologia: Reflexão e Crítica, 20(3), 376-388. doi: 10.1590/So102-79722007000300005

Coelho, H. M. B., Peres, R. S., \& Oliveira, F. S. (2005). A clínica-escola em sua dupla função: usuários e estagiários. In L. L. Melo-Silva, M. A. Santos \& C. P. Simon (Orgs.), Formação em psicologia: serviçosescola em debate (pp. 197-220). São Paulo, SP: Vetor.

Cunha, T. R. S., \& Benetti, S. P. C. (2009). Caracterização da clientela infantil numa clínica-escola de psicologia. Boletim de Psicologia, 59(130), 117-127.

Gatti, A. L., \& Beres, V. L. G. (2004). Queixas em serviço de atendimento psicológico. Integração, 1O(38), 281-284.

Graminha, S. S. V., \& Martins, M. A. O. (1993). Estudo das características da população que procura o serviço de atendimento infantil no Centro de Psicologia Aplicada da FFCLRP-USP. Psico, 24(1), 119-130.

Lopes, G. P., Silva, L. V., \& Vagostello, L. (2013). Caracterização das demandas de psicodiagnóstico infantil em uma clínica-escola de São Paulo (201O-2012) (Trabalho de Conclusão de Curso). Faculdade de Ciências Humanas e Sociais, Universidade São Judas Tadeu, São Paulo.

Macedo, M. M. K., Baldo, M. A., Santos, R. L., Ribas, R. F., Silva, S. M., \& Gonçalves, T. G. (2011). Motivos de busca de atendimento psicológico por adolescentes em uma clínica-escola. Psicologia: Teoria e Prática, 13(2), 63-75. Recuperado de http://pepsic.bvsalud.org/scielo. php?script=sci_arttext\&pid=S1516-36872011000200005\&lng=pt \&tlng $=\mathrm{pt}$ 
Mantovani, C. C. P., Marturano, E. M., \& Silvares, E. F. M. (2010, julho/ setembro). Abandono do atendimento em uma clínica-escola de psicologia infantil: variáveis associadas. Psicologia em estudo, 15(3), 527-535. doi: 10.1590/S1413-73722010000300010

Maravieski, S., \& Serralta, F. B. (2011). Características clínicas e sociodemográficas da clientela atendida em uma clínica-escola de Psicologia. Temas em Psicologia, 19(2), 481-490. Recuperado de http://pepsic.bvsalud.org/scielo.php?script=sci_arttext\& pid=S1413-389X2011000200011\&lng=pt\&tlng=pt

Melo, S. A., \& Perfeito, H. C. C. S. (2006, julho/setembro). Características da população infantil atendida em triagem no período de 2000 a 2002 numa clínica-escola. Estudos de psicologia, 23(3), 239-249. doi: 10.1590/So103-166X2006000300003

Melo-Silva, L. L, Santos, M. A., \& Simon, C. P. (2005). Centro de Pesquisa e Psicologia Aplicada da Faculdade de Filosofia, Ciências e Letras de Ribeirão Preto: formando o psicólogo do futuro. In L. L. MeloSilva, M. A. Santos \& C. P. Simon (Orgs.), Formação em psicologia: serviços-escola em debate (pp. 221-256). São Paulo, SP: Vetor.

Merg, M. M. G. (2008). Características da clientela infantil em clínicasescola (Dissertação de mestrado). Faculdade de Psicologia, Pontifícia Universidade Católica do Rio Grande do Sul, Rio Grande do Sul. Recuperado de http://repositorio.pucrs.br:8080/dspace/ handle/10923/4972

Moura, C. B., Marinho-Casanova, M. L., Meurer, P. H., \& Campana, C. (2008, janeiro/junho). Caracterização da clientela pré-escolar de uma clínicaescola brasileira a partir do Child Behavior Checklist (CBCL). Contextos Clínicos, 1(1), 1-8. Recuperado de http://pepsic.bvsalud.org/scielo. php?script=sci_arttext\&pid=S1983-34822008000100001\&lng=pt \&tlng $=\mathrm{pt}$

Oliveira, M. S., Pereira, R. F., Peixoto, A. C. A, Rocha, M. M., OliveiraMonteiro, N. R., Macedo, M. M. K., \& Silvares, E. F. M. (2014, abril/junho). Supervisão em serviços-escola de psicologia no Brasil: perspectivas dos supervisores e estagiários. Psico, 45(2), e1-e9. doi: 10.15448/1980-8623.2014.2.15417 
Rodrigues, M. C., Campos, A. P. S., \& Fernandes, I. A. (2012, abril/junho). Caracterização da queixa escolar no Centro de Psicologia Aplicada da Universidade Federal de Juiz de Fora. Estudos de Psicologia, 29(2), 241-252. doi: 10.1590/So103-166X2012000200010

Romaro, R. A., \& Capitão, C. G. (2003). Caracterização da clientela da clínica-escola de psicologia da Universidade São Francisco. Psicologia: Teoria e Prática, 5(1), 111-121. Recuperado de http:// editorarevistas.mackenzie.br/index.php/ptp/article/view/1185

Sales, J. R. (1989). Estudo sobre a clientela da área de saúde mental em Varginha. Psicologia, Ciência e Profissão, 9(2), 22-26. doi: 10.159o/ S1414-98931989000200009

Santa-Rosa, E. J. (2008). A família e a criança no psicodiagnóstico infantil: estudo em uma clínica-escola em São Paulo (Tese de doutorado, Pontifícia Universidade Católica de São Paulo, São Paulo). Recuperado de https://sapientia.pucsp.br/handle/handle/17261

Scortegagna, P., \& Levandowski, D. C. (2004, julho/dezembro). Análise dos encaminhamentos de crianças com queixa escolar da rede municipal de ensino de Caxias do Sul. Interações, 9(18), 127-152. Recuperado de http://pepsic.bvsalud.org/scielo.php?script=sci_arttext\& pid $=$ S1413-29072004000200008\&lng $=$ pt\&tlng $=p t$

Wielewicki, A. (2011). Problemas de comportamento infantil: importância e limitações de estudos de caracterização em clínicas-escola Brasileiras. Temas em Psicologia, 19(2), 379-389. Recuperado de http://pepsic. bvsalud.org/scielo.php?script=sci_arttext\&pid=S1413-389X201100 $0200003 \& \operatorname{lng}=\mathrm{pt} \& \operatorname{lng}=\mathrm{pt}$ 